\title{
The influence of pasteurization and starter culture on methanol content and bio-profile of fermented Morinda citrifolia Linn. (Noni) fruit juice
}

\author{
Manee SAELEE ${ }^{1,2}$, Bhagavathi Sundaram SIVAMARUTHI², Sasithorn SIRILUN ${ }^{2}$, Jakkapan SIRITHUNYALUG ${ }^{2}$, \\ Sartjin PEERAJAN ${ }^{3}$, Chaiyavat CHAIYASUT ${ }^{2 \star}$ (D)
}

\begin{abstract}
Morinda citrifolia L., generally known as noni, is commonly used in Thai medicines and cuisine. Several bioactive phytochemicals have been isolated and identified from the noni plant, and their compositions differ based on the cultivars and harvesting period. Different types of fermented noni fruit juices (FNJ) were used as health supplement in several southeastern countries. The formation of a large amount of alcohol, especially methanol, in FNJ is one of the major hindrances to FNJ production. The current study explains the impact of starter culture (Lactobacillus plantarum SK15), pasteurization and addition of EGCG on alcohol content, bioactivity, and the physiochemical property of FNJ. The $\mathrm{pH}$ and sugar content of the samples were reduced during fermentation, while the organic acid content increased. The samples with EGCG obviously exhibited high total phenolic content and antioxidant activity. The level of pectin was high, and pectin methylesterase (PME) activity was low in EGCG-added samples compared with other samples. The alcohol content of the samples was under the permissible level of Thai standard; specifically, the methanol level was low in EGCG-added samples. Pasteurization and addition of starter culture did not influence any of the studied parameters. About $70 \%$ of acceptability index was observed for FNJ samples in the sensory evaluation. The study concluded that the addition of EGCG effectively reduced the alcohol content and improved the quality of FNJ. Also, further studies are required to reveal the role of starter culture in noni fruit fermentation.
\end{abstract}

Keywords: Morinda citrifolia L.; methanol; antioxidant; EGCG; phenolic content; fermentation.

Practical Application: The production of enriched bioactive fermented noni fruit juice with low alcohol content.

\section{Introduction}

Morinda citrifolia L. belongs to the Rubiaceae family and is generally indigenous to tropical regions. M. citrifolia $\mathrm{L}$. is commonly called noni, and almost all parts of the plant have been used in Thailand. For example, noni leaves were used in Thai cuisine, such as hor mok (Wattanathorn et al., 2018). About 200 phytochemicals were isolated and identified from various parts of the noni plant, and the phytochemical composition greatly varied in accordance with the cultivating region and harvesting time (Abou Assi et al., 2017). The phytochemical constituents, such as carbohydrates, soluble protein, phenolic compounds, rutin, and ascorbic acid, varied at the ripening stage of the fruits (Lewis Lujan et al., 2014). A detailed study on the phytochemical constitution of noni fruits, leaves, and root was carried out recently (Abou Assi et al., 2017), but the complete list of the phytochemical contents of noni plant has not yet been reported. Noni plant is recognized due to its commercial (preservative, noni juice, insecticide) and pharmacological (antimicrobial, antioxidant, anti-cancer, anti-inflammation, antidiabetic, anti-tuberculosis, analgesic, anxiolytic, wound healing, anti-psoriasis healing, and immune-enhancing activities) importance (Abou Assi et al., 2017).

Fruit juices are one of better sources of functional components like prebiotics, non-digestible food ingredients that modulate gut microbiota. The prebiotic-rich fruit juices can be prepared either by adding purified prebiotics (like inulin, galactoligosaccharides, fructooligosaccharides, polydextrose, isomaltodextrin, resistant starch) or turn the fruit sugar into prebiotic carbohydrates. The production process significantly influences the quality of fruit juices. Specifically, high temperature and $\mathrm{pH}$ potentially reduce the prebiotic ability of the fruit juice (Fonteles \& Rodrigues, 2018).

As per the market analysis research forecasting, noni fruit juice market is expected to reach significant growth in North America, other than Asia Pacific countries due to the increased demand for preventing health products. The noni fruit juices are made from fresh or decomposed fruits, or by fermentation. The phytochemical content of the fruit juice depends on the extraction method and cultivars. The phytochemical content of Indian fermented noni fruit juice was very different from those of Thai fermented noni juice and American noni fruit juice (Nandhasri et al., 2005; Satwadhar et al., 2011). Noni fruit juice has been recognized as a potent probiotic drink harboring and supporting the survival of probiotics strains such as Lactobacillus spp. and Bifidobacterium spp. (Wang et al., 2009). During fermentation, some undesirable metabolites will form in the fermented juice; for example, alcohol formation, specifically methanol, takes place. Previously, we reported that pasteurization 
reduced the methanol formation during the production of fermented noni fruit juice (FNJ) (Chaiyasut et al., 2013).

The objective of the current study is to determine the impact of addition of epigallocatechin gallate (EGCG), a potent inhibitor of pectin methylesterase (PME) (Lewis et al., 2008), and starter culture as well as sterility of raw materials on methanol content and bioactivity of FNJ.

\section{Materials and methods}

\subsection{Materials}

M. citrifolia L. was obtained from the local market of Sunkampang, Chiang Mai, Thailand, and species confirmation was done with the help of herbarium specimen (Voucher number 023238) of the Faculty of Pharmacy, Chiang Mai University. Methanol and butanol were purchased from Sigma-Aldrich, USA. Ethanol was purchased from Dr. Ehrenstorfer GmbH, Germany. EGCG was purchased from Xian Haoxuan Bio-Tech Co. Ltd. China. The starter culture, Lactobacillus plantarum SK15, was provided by Innovation Center for Holistic Health, Nutraceuticals, and Cosmeceuticals, Faculty of Pharmacy, Chiang Mai University, Thailand. The gas chromatography column, Carbowax 20M polyethylene glycol capillary column $(30 \mathrm{~m} \times 0.53 \mathrm{~mm})$, was purchased from Ohio Valley Specialty, USA.

\subsection{Starter culture preparation}

L. plantarum SK15 was used as a starter in this study. L. plantarum was cultured in de Man, Rogosa and Sharpe (MRS) broth and incubated at $37 \pm 2{ }^{\circ} \mathrm{C}$ for $24 \mathrm{~h}$, to obtain an approximate cell concentration of $10^{9} \mathrm{CFU} / \mathrm{ml}$. About $10 \%$ inoculum was used for the M. citrifolia L. fruit fermentation.

\subsection{Fermentation process}

The nearly ripe $M$. citrifolia $\mathrm{L}$. fruits were washed with sterile (distilled) water, cut into small pieces and blended by a blender (YC112M-4, China). The blended M. citrifolia L. was separated into two groups: pasteurized and non-sterilized (control) groups. Each group was sub-divided into those with and without EGCG $(3.75 \mathrm{mg} / \mathrm{mL})$ and starter $(10 \%)$. The factorial designs for $2^{3}$ experimental variables are shown in Table 1. The experimental groups were coded as follow:

Table 1. The independent variables of $2^{3}$ factorial in completely randomized design (CRD).

\begin{tabular}{cccc}
\hline \multicolumn{4}{c}{ Independent variables } \\
\hline Std & Sterilization methods & EGCG $(\mathrm{mg} / \mathrm{mL})$ & Starter \\
\hline 1 & Pasteurization & 0 & + \\
2 & Non-sterile & 0 & + \\
3 & Pasteurization & 3.75 & + \\
4 & Non-sterile & 3.75 & + \\
5 & Pasteurization & 0 & - \\
6 & Non-sterile & 0 & - \\
7 & Pasteurization & 3.75 & - \\
8 & Non-sterile & 3.75 & - \\
\hline
\end{tabular}

+ Presence of starter; - Absence of starter.

\author{
NEY: Non-sterile + EGCG + Starter culture \\ NEN: Non-sterile + EGCG + No starter culture \\ NY: Non-sterile + Starter culture \\ NN: Non-sterile + No starter culture \\ PEY: Pasteurized + EGCG + Starter culture \\ PEN: Pasteurized + EGCG + No starter culture \\ PY: Pasteurized + Starter culture \\ PN: Pasteurized + No starter culture
}

The fermentation processes were carried out for three different durations such as short term process (up to $24 \mathrm{~h}$ ), medium-term process (up to $72 \mathrm{~h}$ ), and long term process (up to $600 \mathrm{~h}$ ). The blended $M$. citrifolia L. fruits, water, and sugar were mixed at the ratio of 3:10:1 (27.8 g: $92.8 \mathrm{ml}: 9.2 \mathrm{~g})$ and $13 \mathrm{ml}$ of starter culture $\left(10^{9} \mathrm{CFU} / \mathrm{ml}\right.$ stock) (Chaiyasut et al., 2013), and the fermentation process was conducted at $30^{\circ} \mathrm{C}$ and samples were collected at different time points to assess the methanol content.

\subsection{Determination of methanol and ethanol}

Samples were filtered through a 0.22 um nylon membrane filter and mixed with $\mathrm{n}$ - butanol (50 ppm) (internal standard). The amounts of methanol and ethanol in the samples were determined by gas chromatography (GC-14B, Shimadzu, Japan) with Carbowax $20 \mathrm{M}$ polyethylene glycol capillary column $(30 \mathrm{~m} \times 0.53 \mathrm{~mm})$. The flow rate of the nitrogen carrier gas was set at $40 \mathrm{ml} / \mathrm{min}$. The temperatures at the injector port, column oven, and detector were set at 180,38 , and $260^{\circ} \mathrm{C}$ respectively, and splitless injection was set at $5 \mu \mathrm{L}$ for each injection (Chaiyasut et al., 2017a).

\subsection{Determination of total phenolic content}

The total phenolic content of fermented noni juice samples was determined by Folin-Ciocalteu colorimetric method as described previously (Pengkumsri et al., 2015). The values were represented as mg gallic acid equivalent (GAE) per ml of the sample.

\subsection{Antioxidant capacity}

ABTS (2,2'-azino-bis-3-ethylbenzthiazoline-6-sulphonic acid) and DPPH (1,1-diphenyl-2-picryl-hydrazil) based scavenging modules were employed to determine the antioxidant capacity of noni fruit juice at different stages of fermentation as detailed previously (Sivamaruthi et al., 2016). The results were represented as mg trolox equivalents (TE) antioxidant capacity per ml of sample.

\section{$2.7 \mathrm{pH}$ and organic acid content}

The $\mathrm{pH}$ and organic acid content (lactic acid and acetic acid) of fermented noni fruit samples were measured using $\mathrm{pH}$ meter (Metrohm 691) and high-performance liquid chromatography (HPLC) respectively, as detailed previously (Peerajan et al., 2016; Chaiyasut et al., 2017b). The total acidity of the samples was represented by their lactic acid equivalent per $\mathrm{ml}$. The samples were titrated with $0.0940 \mathrm{~N}$ sodium hydroxide using phenolphthalein as indicator (Fabro et al., 2006). 


\subsection{Determination of pectin content and pectin methylesterase (PME) activity}

The pectin content and PME activity of the samples were determined as described in our previous report (Chaiyasut et al., 2013).

\subsection{Determination of sugar content}

The total sugar and reducing sugar contents of the noni samples were measured by phenol-sulfuric acid method and DNS (Dinitrosalicylic acid) method as detailed previously (Masuko et al., 2005; Sirilun et al., 2017).

\subsection{Enumeration of microorganisms}

The total bacterial count (TBC), lactic acid bacteria (LAB), fungi, and representative pathogen load (Bacillus cereus, Escherichia coli, Staphylococcus aureus, and Salmonella spp.) of the fermented noni fruit juices were estimated by serial dilution and plate count method as detailed previously (Sirilun et al., 2016). Specific media, such as Phenol Red Egg-yolk Agar, Eosin-Methylene Blue (EMB) agar, Mannitol Salt Egg-yolk (MSEY) agar, Salmonella-Shigella (SS) agar, and potato dextrose agar, were used for the culturing of specific pathogens. After the appropriate incubation period, colonies were counted, and the colony forming units (CFU) was calculated as follows:

$\mathrm{CFU} / \mathrm{ml}=\frac{(\text { Number of bacterial colonies counted on plate } \times \text { Dilution Factor })}{\text { Volume of culture plate }}$

\subsection{Sensory evaluation of FNJ}

Sensory analysis was performed to assess the acceptability of FNJ in terms of color, sourness, acrid odor, transparency, and overall acceptance. Twenty healthy volunteers (15 women, 5 men, age 21-50 years) participated in the study. The samples were packed in clear tube and coded. The score sheet contained a five-point hedonic scale (Nduko et al., 2018), with the key words rating as following, Dislike very much (1), dislike (2), neither like nor dislike (3), like (4), and like very much (5) were used. The acceptability index was calculated (Lafarga et al., 2019).

\subsection{Statistical analysis}

The experiments were carried out in triplicate. The amount of methanol at each fermentation time was analyzed by one-way ANOVA using the statistical SPSS software version 17 (SPSS Inc., Chicago, IL, USA). Duncan's new multiple range test determined the significant differences at the 95\% confidence level $(p<0.05)$. The values were represented as the mean \pm standard deviation.

\section{Results and discussion}

\subsection{Ph, sugar content and organic acid content of Fermented Noni Juice (FNJ)}

The $\mathrm{pH}$ value of the FNJ was gradually reduced during the fermentation process. The samples with starter culture showed slightly low $\mathrm{pH}$ values (3.26-3.32) compared with non-starter groups (3.85-3.99). However, the sterilization process did not significantly affect the $\mathrm{pH}$ values of the samples. Also, the addition of ECGC did not affect the $\mathrm{pH}$ of the samples (Figure S1 - Supplementary Material). The results suggest that the addition of LAB starter culture significantly reduced the $\mathrm{pH}$ of the fermented product.

The reducing sugar content of the FNJ samples did not show a steady pattern. In the presence of starter culture, the reducing sugar value was reduced from starting point except in the NEY group, while in the absence of starter culture, the value of the reducing sugar did not exhibit a constant trend (Figure S2). The total sugar content was gradually reduced during the fermentation process, and the reduction was instant (drastic reduction was observed after $120 \mathrm{~h}$ of fermentation compared with other samples) in samples with a starter culture (Figure S3). The results suggest that the sterilization process and addition of starter culture significantly influenced the reducing sugar content of the samples, while EGCG did not play an influential role. The fermentation of noni fruit by L. plantarum or Bifidobacterium longum reduced the $\mathrm{pH}$, acidity, and sugar content (Wang et al., 2009), while L. casei failed to survive in the $\mathrm{pH}$ condition. In the present study, L. plantarum SK15 reduced the $\mathrm{pH}$ of $\mathrm{FNJ}$ and survived in low $\mathrm{pH}$ environment.

The lactic acid content of the samples was increased during fermentation, especially in the presence of starter culture in both non-sterile control $(0.09 \pm 0.03-1.01 \pm 0.05)$ and pasteurized $(0.09 \pm 0.02-1.38 \pm 0.08)$ samples. The addition of EGCG did not affect the lactic acid load of FNJ (Figure S4). It is known that the presence of LAB in fermentation medium increases the lactic acid content by its metabolic activities. However, the acetic acid content was not affected by the addition of starter culture, but EGCG significantly affected the acetic acid content in the pasteurized samples. The non-sterile samples displayed an uneven pattern of acetic acid content during fermentation, possibly due to the uncontrolled metabolic activities of natural fermentation (Figure S5). Total acidity (lactic acid equivalent) was gradually increased throughout the fermentation period in all the samples, but the percentage of acidity was higher in samples with a starter culture, which was attributed to the presence of lactic acid bacteria. EGCG did not play any role in the total acidity of the samples (Figure S6).

\subsection{Total phenolic content and antioxidant capacity}

The total phenolic content and antioxidant capacity (ABTS and DPPH) were obviously higher in samples with EGCG, since EGCG is a potent antioxidant. The presence of starter culture slightly influenced the total phenolic content and antioxidant capacity of the samples irrespective of sterility. The phenolic content was increased during the fermentation process. After 25 days of fermentation, NEY and PEY samples showed $1.61 \pm 0.28$ and $1.58 \pm 0.11 \mathrm{mg} \mathrm{GAE}$ per $\mathrm{ml}$ of sample respectively. However, NEN and PEN samples showed $1.41 \pm 0.17$ and $1.33 \pm 0.11 \mathrm{mg}$ GAE per $\mathrm{ml}$ of sample respectively. Nevertheless, the changes were not statistically significant (Figure 1). Samples with EGCG exhibited high antioxidant capacity (measured by DPPH and ABTS assays) compared with other samples in both non-sterile control and pasteurized samples. A non-significant variation was observed between the starter and non-starter samples (Figure 2 and 3). 


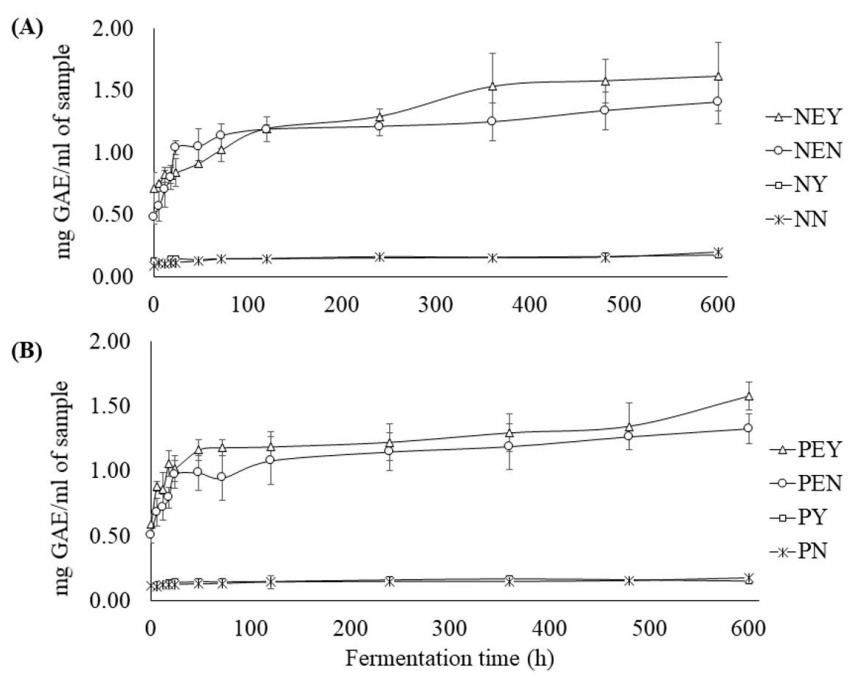

Figure 1. The changes in the total phenolic content of noni fruit juice during fermentation (A) Non-sterile control samples (B) pasteurized samples. NEY: Non-sterile + EGCG + Starter culture; NEN: Non-sterile + EGCG + No starter culture; NY: Non-sterile + Starter culture; $\mathrm{NN}$ : Non-sterile + No starter culture; PEY: Pasteurized + EGCG + Starter culture; PEN: pasteurized + EGCG + No starter culture; PY: Pasteurized + Starter culture; PN: Pasteurized + No starter culture.

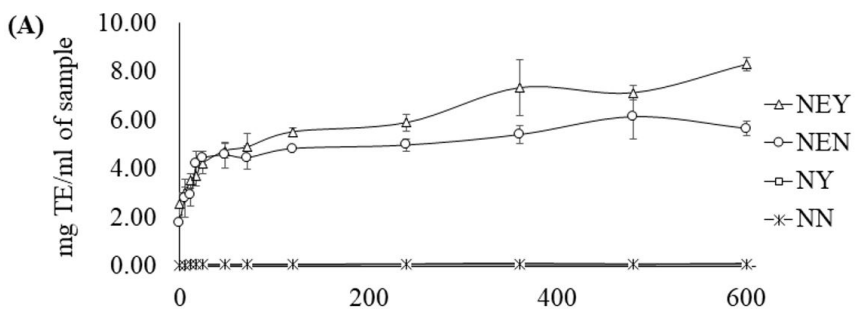

(B)

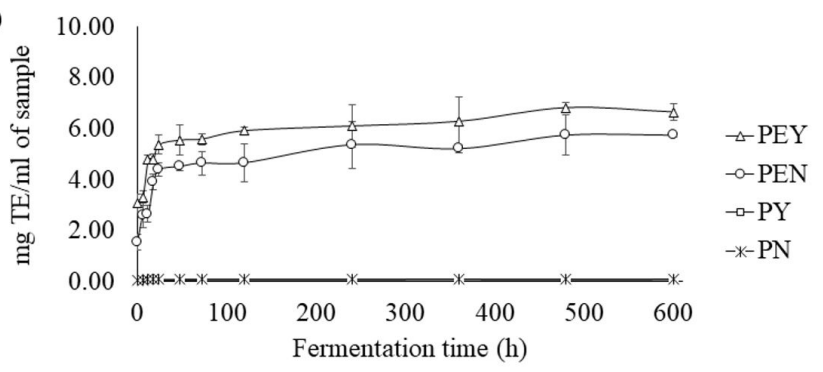

Figure 2. Free-radical scavenging activity (ABTS assay) of noni fruit during fermentation (A) Non-sterile control samples (B) pasteurized samples. NEY: Non-sterile + EGCG + Starter culture; NEN: Non-sterile + EGCG + No starter culture; NY: Non-sterile + Starter culture; NN: Non-sterile + No starter culture; PEY: Pasteurized + EGCG + Starter culture; PEN: pasteurized + EGCG + No starter culture; PY: Pasteurized + Starter culture; PN: Pasteurized + No starter culture.

The lactobacillus-mediated fermentation process reduced the total antioxidant capacity and radical scavenging activity of FNJ, while B. longum increased the antioxidant capacity of FNJ (Wang et al., 2009). The results of the present study suggest that the addition of starter culture does not affect the antioxidant capacity and total phenolic content of FNJ, and the changes in the antioxidant capacity and phenolic content are solely attributed

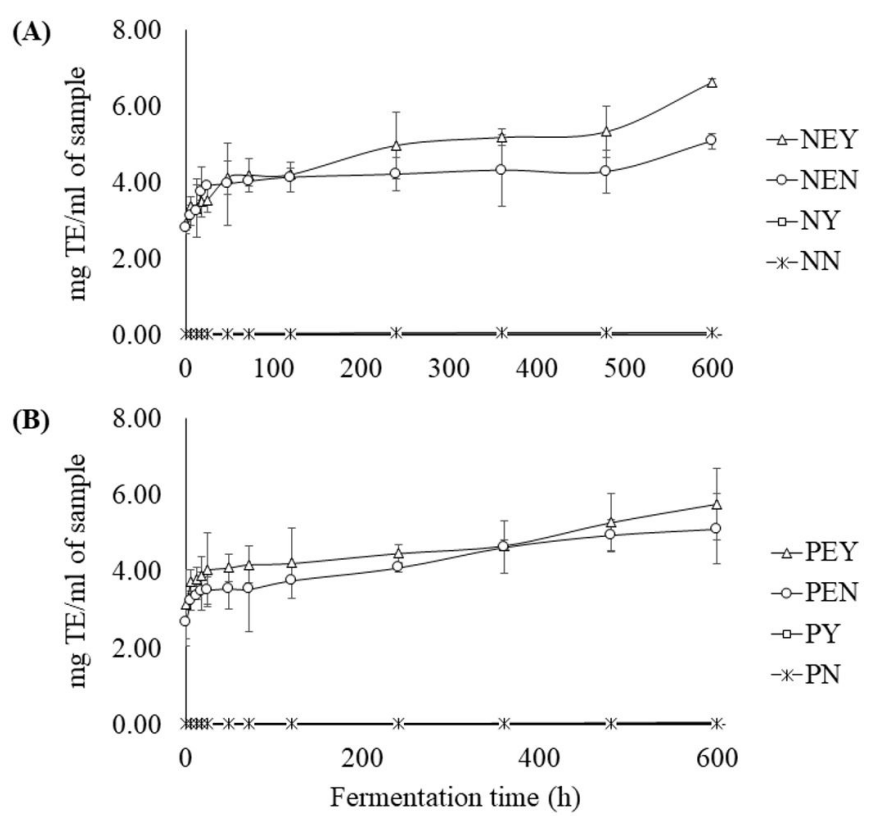

Figure 3. Free-radical scavenging activity (DPPH assay) of noni fruit during fermentation. (A) Non-sterile control samples (B) pasteurized samples. NEY: Non-sterile + EGCG + Starter culture; NEN: Non-sterile + EGCG + No starter culture; NY: Non-sterile + Starter culture; NN: Non-sterile + No starter culture; PEY: Pasteurized + EGCG + Starter culture; PEN: pasteurized + EGCG + No starter culture; PY: Pasteurized + Starter culture; PN: Pasteurized + No starter culture.

to the presence of EGCG. Also, the sterility of the raw material (noni fruit) did not significantly affect the phenolic content and free radical scavenging capability of FNJ.

\subsection{Pectin content and PME activity}

The pectin content was reduced during fermentation in all the samples. The level of pectin was drastically reduced during the initial period of fermentation, but after 4 days of fermentation, the pectin level was increased, possibly due to the digestion of raw materials and release of pectin to the medium. A maximum of $1.61 \pm 0.15 \mathrm{mg} / \mathrm{ml}$ of pectin was noted in PEY sample after $360 \mathrm{~h}$ of fermentation, whereas in PY sample, about $1.57 \pm 0.18 \mathrm{mg} / \mathrm{ml}$ of pectin was observed after $480 \mathrm{~h}$ of fermentation. The results indicate that the presence of EGCG influences the pectin content during the fermentation process. However, after complete fermentation, the level of pectin was found to be in the range of $0.36-0.50 \mathrm{mg} / \mathrm{ml}$ for all samples irrespective of the presence of EGCG and starter culture. The range does not show significant difference among the samples. In the control samples, the pectin content was significantly reduced in non-starter samples (NEN and NN) compared with starter samples (NEY and NY), and NEN $(0.46 \pm 0.04 \mathrm{mg} / \mathrm{ml})$ samples had relatively high pectin content compared with NN $(0.09 \mathrm{mg} / \mathrm{ml})$ samples (Figure $\mathrm{S} 7)$.

The PME activity was significantly low in the EGCG containing samples compared with other samples in both control and pasteurized groups. The PME activity of PEY and PEN samples were $10.58 \pm 0.81$ and $7.35 \pm 1.61 \mu \mathrm{mol} / \mathrm{ml} / \mathrm{min}$ respectively. 
The PME activity of PY and PN samples were $42.70 \pm 4.21$ and $47.31 \pm 3.87 \mu \mathrm{mol} / \mathrm{ml} / \mathrm{min}$ respectively. The difference between PEY and PEN samples as well as between PY and PN samples were not statistically significant. However, PEY showed significant difference in PME activity compared with PY $(p=0.00)$ and PN $(p=0.00)$ samples. The results suggest that the addition of EGCG, obviously, significantly reduced PME activity, and the addition of starter culture did not influence PME activity. In the control samples (NEY, NEN, NY, and NN), the values of PME activity were $8.30 \pm 0.27,8.68 \pm 0.81,37.67 \pm 4.16$, and $42.33 \pm 3.23 \mu \mathrm{mol} / \mathrm{ml} / \mathrm{min}$ respectively (Figure S8).

The addition of chemical enzyme inhibitors or application of physical stress, such as pressure and temperature, may help to reduce or inactivate PME activity. The addition of crude pectin esterase inhibitor from Jelly-Fig significantly reduced the methanol content in Averrhoa carambola L. wine production (Wu et al., 2005). High-pressure processing is an alternative method to inactivate enzymes and microbes in fruits (Aghajanzadeh \& Ziaiifar, 2018). During low temperature-pressure treatment, due to the covalent bonding, the nutritional value and quality of the fruits are not often affected (Polydera et al., 2004, Welti-Chanes et al., 2009). However, during pressure and temperature treatment, denaturation or change of protein active site will deactivate enzyme activity, and these effects may be reversible or not (Aghajanzadeh \& Ziaiifar, 2018).

Chaiyasut et al. (2013) reported that pasteurization significantly inactivated the PME activity in noni fermentation process compared with chemical treatment using potassium metabisulfite. During processing, diced or blended samples showed a significant difference in pectin and PME activity. The study concluded that the pasteurized blended noni fruit samples exhibited low methanol content and low PME activity during fermentation (Chaiyasut et al., 2013). The pectin content was high in the pasteurized samples compared with potassium metabisulfite treated samples (Chaiyasut et al., 2013).

In the present study, EGCG-added samples showed potent inactivation of PME activity and high pectin content (Figure S7 and S8). The addition of starter culture did not significantly influence the pectin content and PME activity in both control and pasteurized groups.

\subsection{Microbial load in FNJ}

The total bacterial load of PEY, PEN, PY, and PN samples after 25 days of fermentation were $5.13 \pm 0.42,2.03 \pm 0.01,5.42 \pm 0.35$, and $2.90 \pm 0.05 \log$ CFU per ml of sample respectively. Similarly, the total bacterial load of NEY, NEN, NY, and NN samples after 25 days of fermentation were $5.57 \pm 0.41,2.90 \pm 0.02$, $5.80 \pm 0.37$, and $2.88 \pm 0.02 \log$ CFU per ml of sample respectively. The LAB count of PEY, PEN, PY, and PN samples after 25 days of fermentation were $4.87 \pm 0.14,1.50 \pm 0.02,5.36 \pm 0.27$, and $2.27 \pm 0.01 \log$ CFU per $\mathrm{ml}$ of sample respectively. In addition, the LAB count of NEY, NEN, NY, and NN samples after 25 days of fermentation were $5.00 \pm 0.20,2.77 \pm 0.06,5.57 \pm 0.25$, and $2.31 \pm 0.06 \log$ CFU per $\mathrm{ml}$ of sample respectively. The tested pathogenic bacterial strains, yeast and fungus, were not detected in any of the samples at any point of analysis, which suggest that the FNJ was free from pathogenic microbial contamination. The results showed no significant difference between non-sterile and pasteurized samples in terms of microbial load. The strong antimicrobial property of the noni itself prevented contamination during the fermentation process (Figure S9 and S10). Generally, pasteurization removes all the pathogenic microbes residing on the raw materials. In addition, due to the strong anti-microbial activity of noni fruit (Abou Assi et al., 2017), FNJ was not contaminated with any pathogen during the fermentation process.

\subsection{Methanol and ethanol content of FNJ}

The methanol content of NEY, NEN, NY, and NN samples were $73.70 \pm 8.94,92.03 \pm 6.58,252.13 \pm 6.96$, and $288.41 \pm 16.70 \mathrm{ppm}$ respectively after $600 \mathrm{~h}$ of fermentation. However, PEY, PEN, PY, and $\mathrm{PN}$ samples contained $56.05 \pm 8.66,48.22 \pm 4.48,198.52 \pm 19.11$, and $296.56 \pm 21.31 \mathrm{ppm}$ of methanol after $600 \mathrm{~h}$ of fermentation respectively. The presence of EGCG and starter culture relatively reduced the methanol content in FNJ compared with other samples. The addition of EGCG significantly reduced the methanol content in both non-sterile control and pasteurized samples. The pasteurization process significantly reduced the methanol content of the pasteurized samples compared with their non-sterile counterparts (Figure 4).

The ethanol content of noni fruit juice was reduced after fermentation. The addition of starter culture and EGCG significantly influenced the ethanol content of FNJ. In the control

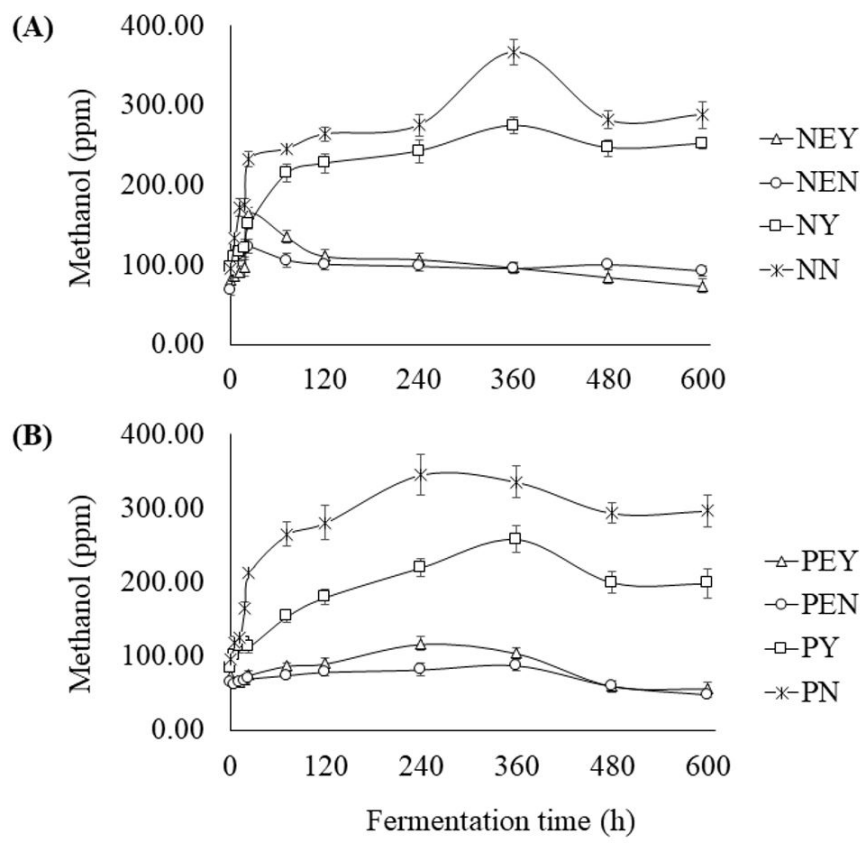

Figure 4. The methanol content of noni fruit juice during fermentation. (A) Non-sterile control samples (B) pasteurized samples. NEY: Non-sterile + EGCG + Starter culture; NEN: Non-sterile + EGCG + No starter culture; NY: Non-sterile + Starter culture; NN: Non-sterile + No starter culture; PEY: Pasteurized + EGCG + Starter culture; PEN: pasteurized + EGCG + No starter culture; PY: Pasteurized + Starter culture; PN: Pasteurized + No starter culture. 


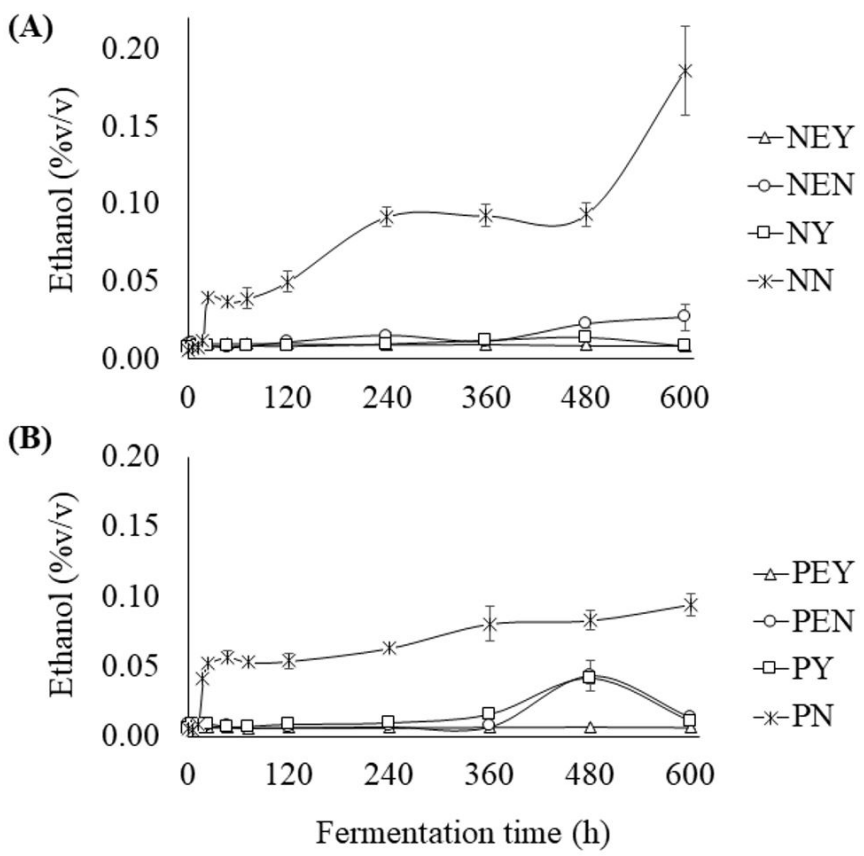

Figure 5. The ethanol content of noni fruit juice during fermentation. (A) Non-sterile control samples (B) pasteurized samples. NEY: Non-sterile + EGCG + Starter culture; NEN: Non-sterile + EGCG + No starter culture; NY: Non-sterile + Starter culture; NN: Non-sterile + No starter culture; PEY: Pasteurized + EGCG + Starter culture; PEN: pasteurized + EGCG + No starter culture; PY: Pasteurized + Starter culture; PN: Pasteurized + No starter culture.

samples, the addition of EGCG did not affect the ethanol content significantly, while starter culture plays a critical role (Figure 5).

Several methods have been employed to reduce the methanol content via inactivating the PME activities in citrus fruits. For instance, the addition of phenolic acids $(0.2 \mathrm{mg} / \mathrm{l}$ of gallic or coumaric acid) along with pectic enzyme during wine preparation improved the quality of the wine and reduced the methanol content (Hou et al., 2008). Pasteurization reduced the methanol content in naturally fermented noni fruit juice via inactivating the PME activity (Chaiyasut et al., 2013). High-pressure homogenizing process reduced the PME activity in orange juices (Welti-Chanes et al., 2009).

The results of the current study suggest that the presence of EGCG effectively reduced the methanol and ethanol contents in FNJ. Pasteurization and addition of starter culture did not have a significant role in methanol formation. Based on Thai community product standard (TCPS 481/2004; up to $240 \mathrm{mg} / \mathrm{l}$ of methanol and $3 \%(\mathrm{v} / \mathrm{v})$ ethanol), all the samples including control samples exhibited a permissible level of alcohol content.

\subsection{Sensory analysis}

The sensory analysis of FNJ showed that the color and transparency of the juices were not significantly changed during various treatments. The sour odor, acrid odor scores are significantly varied among the samples. The overall acceptance scores also differed significantly between the samples. PEN, PEY, NEY, NEN samples exhibited high acceptability index of 70, 69, 68, and 67\%, respectively (Table S1). The overall acceptance score of the FNJ samples was significantly diverse among the samples, but the pasteurization process, addition of starter culture, and EGCG have not significantly influenced the acceptability index of the FNJ. The results suggested that use of EGCG, starter culture and pasteurization improved the FNJ quality, which was not necessarily influence the sensory scores.

\section{Conclusion}

In our previous study, we revealed that blended pasteurized noni fruits are suitable for the production of low methanol-containing naturally fermented noni fruit juice (Chaiyasut et al., 2013). The results of the current study revealed that the addition of EGCG effectively reduced the PME activity and methanol content of FNJ. In addition, pasteurization process and use of starter culture (L. plantarum SK15) did not significantly influence the methanol content and other studied parameters. Even though pasteurization can remove microbial contamination and inactivate PME activity in noni, the addition of EGCG greatly influenced the results of the current study. FNJ samples with EGCG exhibited high acceptability index in sensory analysis. Every LAB culture is unique, so further detailed studies using $\mathrm{LAB}$ starter cultures are required to reveal the significance of the use of specific starter culture for the production of FNJ.

\section{Acknowledgements}

MS acknowledge Research and Researcher Development for Industry Program (RRi) of the Thailand Research Fund (TRF) and Graduate School, Chiang Mai University. BSS wish to acknowledge the Chiang Mai University-Post doctoral fellowship for the support. All the authors wish to thank Chiang Mai University-grant (CMU-grant) for the support. We gratefully acknowledge the Faculty of Pharmacy, Chiang Mai University, Thailand.

\section{References}

Abou Assi, R., Darwis, Y., Abdulbaqi, I. M., khan, A. A., Vuanghao, L., \& Laghari, M. H. (2017). Morinda citrifolia (Noni): A comprehensive review on its industrial uses, pharmacological activities, and clinical trials. Arabian Journal of Chemistry, 10(5), 691-707. http://dx.doi. org/10.1016/j.arabjc.2015.06.018.

Aghajanzadeh, S., \& Ziaiifar, A. M. (2018). A review of pectin methylesterase inactivation in citrus juice during pasteurization. Trends in Food Science \& Technology, 71, 1-12. http://dx.doi. org/10.1016/j.tifs.2017.10.013.

Chaiyasut, C., Supakan, J., Chakkrapong, K., SartjinPeerajan, Sasithorn, S., \& Lalida, S. (2013). Factors affecting methanol content of fermented plant beverage containing Morinda citrifolia. African Journal of Biotechnology, 12(27), 4356-4363. http://dx.doi.org/10.5897/ AJB10.1377.

Chaiyasut, C., Makhamrueang, N., Peerajan, S., \& Sivamaruthi, B. S. (2017a). Assessment of organic acid content, and brix value of representative indigenous fermented plant beverages of Thailand. Asian Journal of Pharmaceutical and Clinical Research, 10(1), 350354. http://dx.doi.org/10.22159/ajpcr.2017.v10i1.15455. 
Chaiyasut, C., Sivamaruthi, B., Peerajan, S., Sirilun, S., Chaiyasut, K., \& Kesika, P. (2017b). Assessment of heavy metals, minerals, alcohol, and fusel oil content of selected fermented plant beverages of Thailand. International Food Research Journal, 24(1), 126-133.

Fabro, M. A., Milanesio, H., Robert, L., Speranza, J., Murphy, M., Rodríguez, G., \& Castañeda, R. (2006). Determination of acidity in whole raw milk: comparison of results obtained by two different analytical methods. Journal of Dairy Science, 89(3), 859-861. http:// dx.doi.org/10.3168/jds.S0022-0302(06)72149-X. PMid:16507678.

Fonteles, T. V., \& Rodrigues, S. (2018). Prebiotic in fruit juice: processing challenges, advances, and perspectives. Current Opinion in Food Science, 22, 55-61. http://dx.doi.org/10.1016/j.cofs.2018.02.001.

Hou, C. Y., Lin, Y. S., Wang, Y. T., Jiang, C. M., Lin, K. T., \& Wu, M. C. (2008). Addition of phenolic acids on the reduction of methanol content in wine. Journal of Food Science, 73(5), C432-C437. http:// dx.doi.org/10.1111/j.1750-3841.2008.00768.x. PMid:18576990.

Lafarga, T., Acién-Fernández, F. G., Castellari, M., Villaró, S., Bobo, G., \& Aguiló-Aguayo, I. (2019). Effect of microalgae incorporation on the physicochemical, nutritional, and sensorial properties of an innovative broccoli soup. Lebensmittel-Wissenschaft + Technologie, 111, 167-174. http://dx.doi.org/10.1016/j.lwt.2019.05.037.

Lewis, K. C., Selzer, T., Shahar, C., Udi, Y., Tworowski, D., \& Sagi, I. (2008). Inhibition of pectin methyl esterase activity by green tea catechins. Phytochemistry, 69(14), 2586-2592. http://dx.doi. org/10.1016/j.phytochem.2008.08.012. PMid:18829053.

Lewis Lujan, L. M., Assanga, I., Bernard, S., Rivera-Castañeda, E. G., Gil-Salido, A. A., Acosta-Silva, A. L., \& Rubio-Pino, J. L. (2014). Nutritional and Phenolic composition of Morinda citrifolia L.(Noni) fruit at different ripeness stages and seasonal patterns harvested in Nayarit, Mexico. International Journal of Food Sciences and Nutrition, 3(5), 421-429. http://dx.doi.org/10.11648/j.ijnfs.20140305.19.

Masuko, T., Minami, A., Iwasaki, N., Majima, T., Nishimura, S. I., \& Lee, Y. C. (2005). Carbohydrate analysis by a phenol-sulfuric acid method in microplate format. Analytical Biochemistry, 339(1), 6972. http://dx.doi.org/10.1016/j.ab.2004.12.001. PMid:15766712.

Nandhasri, P., Pawa, K. K., Kaewtubtim, J., Jeamchanya, C., Jansom, C., \& Sattaponpun, C. (2005). Nutraceutical properties of Thai "Yor" Morinda citrifolia and "Noni" juice extract. Songklanakarin Journal of Science and Technology, 27(2), 579-586.

Nduko, J. M., Maina, R. W., Muchina, R. K., \& Kibitok, S. K. (2018). Application of chia (Salvia hispanica) seeds as a functional component in the fortification of pineapple jam. Food Science \& Nutrition, 6(8), 2344-2349. http://dx.doi.org/10.1002/fsn3.819. PMid:30510734.

Peerajan, S., Chaiyasut, C., Sirilun, S., Chaiyasut, K., Kesika, P., \& Sivamaruthi, B. S. (2016). Enrichment of nutritional value of Phyllanthus emblica fruit juice using the probiotic bacterium, Lactobacillus paracase $i$ HII01 mediated fermentation. Food Science and Technology, 36(1), 116-123. http://dx.doi.org/10.1590/1678457X.0064.
Pengkumsri, N., Chaiyasut, C., Saenjum, C., Sirilun, S., Peerajan, S., Suwannalert, P., Sirisattha, S., \& Sivamaruthi, B. S. (2015). Physicochemical and antioxidative properties of black, brown and red rice varieties of northern Thailand. Food Science and Technology, 35(2), 331-338. http://dx.doi.org/10.1590/1678-457X.6573.

Polydera, A. C., Galanou, E., Stoforos, N. G., \& Taoukis, P. S. (2004). Inactivation kinetics of pectin methylesterase of greek Navel orange juice as a function of high hydrostatic pressure and temperature process conditions. Journal of Food Engineering, 62(3), 291-298. http://dx.doi.org/10.1016/S0260-8774(03)00242-5.

Satwadhar, P. N., Deshpande, H. W., Hashmi, S. I., \& Syed, K. A. (2011). Nutritional composition and identification of some of the bioactive components in Morinda citrifolia juice. International Journal of Pharmacy and Pharmaceutical Sciences, 3(1), 58-59.

Sirilun, S., Sivamaruthi, B. S., Kesika, P., Makhamrueang, N., Chaiyasut, K., Peerajan, S., \& Chaiyasut, C. (2017). Development and evaluation of Mustard green pickled liquid as starter for Morinda citrifolia Linn. Fermentation. International Food Research Journal, 24(5), 2170-2176.

Sirilun, S., Sivamaruthi, B. S., Kumar, N., Kesika, P., Peerajan, S., \& Chaiyasut, C. (2016). Lactobacillus-fermented plant juice as a potential ingredient in cosmetics: Formulation and assessment of natural mouthwash. Asian Journal of Pharmaceutical and Clinical Research, 9(9), 52-56. http://dx.doi.org/10.22159/ajpcr.2016.v9s3.14707.

Sivamaruthi, B. S., Pengkumsri, N., Saelee, M., Kesika, P., Sirilun, S., Peerajan, S., \& Chaiyasut, C. (2016). Impact of physical treatments on stability and radical scavenging capacity of anthocyanidins. International Journal of Pharmacy and Pharmaceutical Sciences, 8(1), 162-167.

Wang, C. Y., Ng, C. C., Su, H., Tzeng, W. S., \& Shyu, Y. T. (2009). Probiotic potential of noni juice fermented with lactic acid bacteria and bifidobacteria. International Journal of Food Sciences and Nutrition, 60(Supp. 6), 98-106. http://dx.doi.org/10.1080/09637480902755095. PMid:19340670.

Wattanathorn, J., Thipkaew, C., Thukham-mee, W., Muchimapura, S., Wannanon, P., \& Tong-un, T. (2018). Morinda citrifolia L. Leaf Extract Protects against Cerebral Ischemia and Osteoporosis in an In Vivo Experimental Model of Menopause. Oxidative Medicine and Cellular Longevity, 13, 1039364. http://dx.doi.org/10.1155/2018/1039364. PMid:29765488.

Welti-Chanes, J., Ochoa-Velasco, C. E., \& Guerrero-Beltrán, J. Á. (2009). High-pressure homogenization of orange juice to inactivate pectinmethylesterase. Innovative Food Science \& Emerging Technologies, 10(4), 457-462. http://dx.doi.org/10.1016/j.ifset.2009.05.012.

Wu, J. S. B., Wu, M. C., Jiang, C. M., Hwang, Y. P., Shen, S. C., \& Chang, H. M. (2005). Pectinesterase inhibitor from jelly-fig (Ficus awkeotsang Makino) achenes reduces methanol content in carambola wine. Journal of Agricultural and Food Chemistry, 53(24), 9506-9511. http://dx.doi.org/10.1021/jf0506277. PMid:16302769. 


\section{Supplementary Material}

Supplementary material accompanies this paper.

Figure S1. pH profile of the noni fruit juice during fermentation. (A) Non-sterile control samples (B) pasteurized samples. NEY: Non-sterile + EGCG + Starter culture; NEN: Non-sterile + EGCG + No starter culture; NY: Non-sterile + Starter culture; NN: Non-sterile + No starter culture; PEY: Pasteurized + EGCG + Starter culture; PEN: pasteurized + EGCG + No starter culture; PY: Pasteurized + Starter culture; PN: Pasteurized + No starter culture.

Figure S2. The reducing sugar content of the noni fruit juice during fermentation. (A) Non-sterile control samples (B) pasteurized samples. NEY: Non-sterile + EGCG + Starter culture; NEN: Non-sterile + EGCG + No starter culture; NY: Non-sterile + Starter culture; NN: Non-sterile + No starter culture; PEY: Pasteurized + EGCG + Starter culture; PEN: pasteurized + EGCG + No starter culture; PY: Pasteurized + Starter culture; PN: Pasteurized + No starter culture.

Figure S3. Total sugar content of the noni fruit juice during fermentation. (A) Non-sterile control samples (B) pasteurized samples. NEY: Non-sterile + EGCG + Starter culture; NEN: Non-sterile + EGCG + No starter culture; NY: Non-sterile + Starter culture; NN: Non-sterile + No starter culture; PEY: Pasteurized + EGCG + Starter culture; PEN: pasteurized + EGCG + No starter culture; PY: Pasteurized + Starter culture; PN: Pasteurized + No starter culture.

Figure S4. The lactic acid content of the noni fruit juice during fermentation. (A) Non-sterile control samples (B) pasteurized samples. NEY: Non-sterile + EGCG + Starter culture; NEN: Non-sterile + EGCG + No starter culture; NY: Non-sterile + Starter culture; NN: Non-sterile + No starter culture; PEY: Pasteurized + EGCG + Starter culture; PEN: pasteurized + EGCG + No starter culture; PY: Pasteurized + Starter culture; PN: Pasteurized + No starter culture.

Figure S5. The acetic acid content of the noni fruit juice during fermentation. (A) Non-sterile control samples (B) pasteurized samples. NEY: Non-sterile + EGCG + Starter culture; NEN: Non-sterile + EGCG + No starter culture; NY: Non-sterile + Starter culture; NN: Non-sterile + No starter culture; PEY: Pasteurized + EGCG + Starter culture; PEN: pasteurized + EGCG + No starter culture; PY: Pasteurized + Starter culture; PN: Pasteurized + No starter culture.

Figure S6. Total acidity of the noni fruit juice during fermentation. (A) Non-sterile control samples (B) pasteurized samples. NEY: Non-sterile + EGCG + Starter culture; NEN: Non-sterile + EGCG + No starter culture; NY: Non-sterile + Starter culture; NN: Non-sterile + No starter culture; PEY: Pasteurized + EGCG + Starter culture; PEN: pasteurized + EGCG + No starter culture; PY: Pasteurized + Starter culture; PN: Pasteurized + No starter culture.

Figure S7. The changes in pectin content of noni fruit during fermentation. (A) Non-sterile control samples (B) pasteurized samples. NEY: Non-sterile + EGCG + Starter culture; NEN: Non-sterile + EGCG + No starter culture; NY: Non-sterile + Starter culture; NN: Non-sterile + No starter culture; PEY: Pasteurized + EGCG + Starter culture; PEN: pasteurized + EGCG + No starter culture; PY: Pasteurized + Starter culture; PN: Pasteurized + No starter culture.

Figure S8. Pectin methyl esterase activity in noni fruit juice during fermentation. (A) Non-sterile control samples (B) pasteurized samples. NEY: Non-sterile + EGCG + Starter culture; NEN: Non-sterile + EGCG + No starter culture; NY: Non-sterile + Starter culture; NN: Non-sterile + No starter culture; PEY: Pasteurized + EGCG + Starter culture; PEN: pasteurized + EGCG + No starter culture; PY: Pasteurized + Starter culture; PN: Pasteurized + No starter culture.

Figure S9. The microbial profile of non-sterile control samples during fermentation. (A) NEY: Non-sterile + EGCG + Starter culture; (B) NEN: Non-sterile + EGCG + No starter culture; (C) NY: Non-sterile + Starter culture; (D) NN: Non-sterile + No starter culture.

Figure S10. The microbial profile of pasteurized samples during fermentation. (A) PEY: Pasteurized + EGCG + Starter culture; (B) PEN: pasteurized + EGCG + No starter culture; (C) PY: Pasteurized + Starter culture; (D) PN: Pasteurized + No starter culture.

Table S1. Sensory analysis of fermented M. citrifolia Linn. fruit juice

This material is available as part of the online article from http://www.scielo.br/cta 